PESQUIMAT Revista de la Fac. CC. MM. de la

Universidad Nacional Mayor de San Marcos

Vol. IX No 1, pp. 39 - 62, LIMA - PERÚ. Julio 2006

\title{
DECAIMIENTO DE LA ECUACIÓN DE ONDA CON DISIPACIÓN
}

\section{Yolanda Silva Santiago Ayala ${ }^{1}$ y Luis Cortés Vega ${ }^{2}$}

\begin{abstract}
RESUMEN.- En este trabajo consideramos el problema de existencia de soluciones globales para la ecuación escalar de la onda con disipación. Estudiamos también el comportamiento asintótico de las soluciones, y presentamos un método alternativo inspirado en técnicas no lineales; específicamente usamos el método de Conrad-Rao [1].
\end{abstract}

PALABRAS CLAVE.- Ecuación de onda, decaimiento de solución, método de Conrad-Rao.

ABSTRACT.- In this work, we consider the problem of existence of global solutions for a scalar wave equation with dissipation. We study also, the asymptotic behaviour of the solutions. In this part of the paper, we present an alternative method - inspired in nonlinear techniques. In order to attack this problem, specifically, we used the Conrad - Rao method [1].

KEY WORDS.- Wave equation, decay of the solution, Conrad-Rao method.

\section{INTRODUCCIÓN}

Estudiaremos el siguiente problema de evolución

$$
\begin{aligned}
& u_{t t}-\Delta u+a(x) u_{t}= 0 \text { en } \Omega \times \mathbb{R}^{+}, \\
& u=0 \text { en } \partial \Omega \times \mathbb{R}^{+}, \\
& u(0)=u^{0}, \quad u_{t}(0)=u^{1} .
\end{aligned}
$$

donde $\Omega$ es un conjunto abierto acotado en $\mathbb{R}^{N}$ con frontera suave $\partial \Omega$ y $a$ es una adecuada función no-negativa y suave $(a \geq 0)$ en $\bar{\Omega}$; además, $a$ puede ser nulo en alguna parte de $\bar{\Omega}$.

Definamos por

$$
E(t)=\frac{1}{2} \int_{\Omega}\left|u_{t}\right|^{2}+|\nabla u|^{2} d x
$$

la energía asociada al sistema (1.1) - (1.3). Debido al Lema 4.1 E es no creciente. Así, estamos interesados en saber que sucede con $E(t)$ cuando $t$ va al infinito y cual es la tasa con la cual decae. En este trabajo, estudiamos la existencia de solución global y el comportamiento asintótico, de la ecuación de la onda con disipación, donde era la condición inicial satisface la condición de compatibilidad de m-ésimo orden, la cual nos permite obtener una solución más regular.

Usamos la Teoría de semigrupos para probar la existencia y unidicidad de solución del problema (1.1) - (1.3), también como su dependencia continua del dato inicial.

\footnotetext{
1 Universidad Nacional Mayor de San Marcos. Facultad de Ciencias Matemáticas. e-mail:ysantiagoa@unmsm.edu.pe

${ }^{2}$ Universidad de Antofagasta, Chile.e-mail:1cortes@uantof.cl
} 
Asimismo estudiamos la regularidad de esta solución.

Haciendo uso de técnicas multiplicativas [6], obtenemos importantes estimativas como (4.12), (4.16), (4.23). Por una adecuada adaptación del método de Conrad y Rao [1], obtenemos la estimativa (4.44) la cual nos permite probar el Lema 4.3 y por consiguiente la hipótesis del Lema 2.1.

En otro estudio puede ser visto en Nakao [8].

Algunos tópicos relativos a la ecuación de la onda pueden verse en [9], [10], [11] y [13].

\section{PRINCIPALES RESULTADOS}

Empezamos enunciando el resultado de existencia de solución del problema (1.1) - (1.3).

Teorema 2.1 Dado $\left(u_{0}, u_{1}\right) \in\left(H^{2}(\Omega) \cap H_{0}^{1}(\Omega)\right) \times H_{0}^{1}(\Omega)$, existe únicamente una solución $u(x, t)$ del problema (1.1) - (1.3) en

$C^{2}\left([0, \infty), L^{2}(\Omega)\right) \cap C^{1}\left([0, \infty), H_{0}^{1}(\Omega)\right) \cap C\left([0, \infty), H^{2}(\Omega) \cap H_{0}^{1}(\Omega)\right)$.

También, necesitamos del siguiente resultado de regularidad de solución, para el cual citamos Kesavan [4] y Ikawa [3]. Introducimos la siguiente definición.

Definición 2.1 La condición inicial $\left(u^{0}, u^{1}\right) \in H^{m+1} \times H^{m}$ satisface la condición de compatibilidad de m-ésimo orden asociado a (1.1) - (1.3) si

$$
u^{k} \in H^{m+1-k} \cap H_{0}^{1} \text { para } k=0,1, \ldots ., m \quad y u^{m+1} \in L^{2},
$$

donde la sucesión $\left(u^{k}\right)_{k}$ está definido por inducción desde $\left(u^{0}, u^{1}\right)$ por la fórmula

$$
u^{k+2}=\Delta u^{k}-a(x) u^{k+1}
$$

Proposición 2.1 Sea $m \geq 1$ un entero. Supongamos que $a \in C^{m-1}(\bar{\Omega})$ y $\left(u^{0}, u^{1}\right)$ satisface la condición de compatibilidada de m-ésimo orden asociado a (1.1) - (1.3). Entonces, existe únicamente una solución $u(t)$ del problema (1.1) - (1.3) tal que

$$
u \in X_{m}=\bigcap_{k=0}^{m} C^{k}\left(\mathbb{R}^{+}, H^{m+1-k} \cap H_{0}^{1}\right) \cap C^{m+1}\left(\mathbb{R}^{+}, L^{2}\right)
$$

es continua. i.e. $\exists C>0$ tal que $\sum_{k=0}^{m+1}\left\|D^{k} u(t)\right\|_{L^{2}}^{2} \leq C\left\|\left(u^{0}, u^{1}\right)\right\|_{H^{m+1} \times H^{m}}^{2}$, donde $D^{k}$ denota la diferenciación parcial de $k$-ésimo orden, con respecto a $t$ y $x$.

Supongamos que $\Omega$ sea la bola abierta en $\mathbb{R}^{N}$ centrada en 0 y de radio $\mathrm{R}$. También asumimos que 
$\forall|x| \geq \frac{R}{2}, \quad a(x):=\tilde{a}(|x|)$, donde $\tilde{a}:\left[\frac{R}{2}, R\right] \rightarrow \mathbb{R}^{+}$es una función estrictamente decreciente la cual satisface $\tilde{a}(R)=0$. (Observemos que $\frac{R}{2}$ puede ser reemplazado por cualquier $R-\varepsilon$ con $\varepsilon>0)$. Sea

$$
\forall r \in\left[0, \frac{R}{2}\right], b(r):=\tilde{a}(R-r) \quad y \quad B(r):=r b(r)
$$

Observamos que $B$ es continua y estrictamente creciente en $\left[0, \frac{R}{2}\right]$ y que $B(0)=0$.

También,

$$
b(r) \rightarrow 0=\tilde{a}(R) \quad \text { cuando } r \rightarrow 0 .
$$

Esto es

$$
a(x) \rightarrow 0 \text { cuando } x \rightarrow \partial \Omega \text {. }
$$

Nosotros usaremos el siguiente Lema en la prueba del Teorema Principal

Lema 2.1 Sea $E: \mathbb{R}^{+} \rightarrow \mathbb{R}^{+}$una función no creciente y $\phi: \mathbb{R}^{+} \rightarrow \mathbb{R}^{+}$una función $C^{1}$ estrictamente creciente tal que

$$
\phi(t) \rightarrow+\infty \text { cuando } t \rightarrow+\infty \text {. }
$$

Asumamos que existen $\sigma>=, \sigma^{\prime}>0$ y $c>0$ tal que

$$
\forall s \geq 1, \int_{s}^{+\infty} E(t)^{1+\sigma} \phi^{\prime}(t) d t \leq c E(s)^{1+\sigma}+c \frac{E(s)}{\phi(s)^{\sigma^{\prime}}} .
$$

Entonces existe $C>0$ dependiendo continuamente de $E(1)$ satisfaciendo

$$
\forall t \geq 1, \quad E(t) \leq \frac{C}{\phi(t)^{\frac{\left(1+\sigma^{\prime}\right)}{\sigma}} .}
$$

Prueba.- Ver [12].

El resultado principal de este trabajo es el siguiente:

Teorema 2.2 (Resultado principal) Supongamos que a va para cero en la frontera, lo suficientemente rápido de modo que existen $p>0$ y $C>0$ tal que

$$
\forall s \in\left(0, \frac{R}{2}\right), \quad \int_{s}^{\frac{R}{2}} \frac{1}{b(r)^{p}} d r \leq C \frac{s}{b(s)^{p}}
$$


Sea $m>\frac{N}{2}$. Entonces si $\left(u^{0}, u^{1}\right)$ satisface la condición de compatibilidad de m-ésimo orden; existe $C>0$ la cual depende de la norma de la condición inicial en $H^{m+1}(\Omega) \times H^{m}(\Omega)$ tal que la solución u de (1.1) - (1-3) verifica

$$
E(t) \leq C\left(B^{-1}\left(\frac{1}{2}\right)\right)^{\frac{2 m}{N}}
$$

donde $B^{-1}$ denota la función de $B$.

\subsection{Observaciones del Teorema}

Observación 2.1 La Tesis del Teorema dice que la energía asociada al sistema (1.1) - (1.3) va para cero cuando $t \rightarrow+\infty$. Pues si $t \rightarrow+\infty$ entonces $\frac{1}{t} \rightarrow 0$ y como $B^{-1}$ es creciente entonces $B^{-1}\left(\frac{1}{t}\right) \rightarrow 0$, i.e. $\left(B^{-1}\left(\frac{1}{t}\right)\right)^{\frac{2 m}{N}} \rightarrow 0$.

Observación 2.2 Si $\exists n \in \mathbb{N}$ tal que $n \geq 2$ y $\exists p>0$ tal que $b(r) \leq n r^{\frac{1}{p}} b(n r), \forall r \in\left[0, \frac{R}{2 n}\right]$. Entonces se verifica (2.9).

Observación 2.3 Si $b(r)=r^{k}$ con $k>0$ y $p k>1$, entonces se verifica (2.9).

Por lo tanto, debido al Teorema principal obtenemos

$$
E(t) \leq \frac{C}{t^{\frac{1}{(k+1) \theta}}} .
$$

Observación 2.4 Si $b(r)=\frac{1}{|L n r|}$ entonces (2.9) no es verdad.

Observación 2.5 Si $b(r)=r^{q} e^{-\frac{1}{r^{k}}}$ con $k>0$ entonces, podemos aplicar la observación 2.2. Por lo tanto, debido al Teorema principal conseguimos

$$
E(t) \leq \frac{C}{\left[(\operatorname{Lnt})^{\frac{1}{k}}\right]^{\frac{2 m}{N}}}
$$

\section{EXISTENCIA DE SOLUCIÓN}

De la ecuación (1.1) escribiendo $v=u_{t}$ conseguimos.

$$
\left(\begin{array}{l}
u \\
v
\end{array}\right)_{t}=\left(\begin{array}{c}
u_{t} \\
u_{t t}
\end{array}\right)=\left(\begin{array}{c}
u_{t} \\
\Delta u-a u_{t}
\end{array}\right)=\left(\begin{array}{c}
v \\
\Delta u-a v
\end{array}\right)=\left(\begin{array}{cc}
0 & I \\
\Delta & -a I
\end{array}\right)\left(\begin{array}{l}
u \\
v
\end{array}\right)
$$


definimos el Operador $A: D(A) \subset H \rightarrow H$,

$$
A=\left(\begin{array}{cc}
0 & I \\
\Delta & -a I
\end{array}\right)
$$

donde $H=H_{0}^{1}(\Omega) \times L^{2}(\Omega)$ y $D(A)=H^{2}(\Omega) H_{0}^{1}(\Omega) \times H_{0}^{1}(\Omega)$.

Así (1.1) - (1.3) es equivalente a

$$
P V I \mid \begin{aligned}
& U_{t}(t)=A U(t) \\
& U(0)=U_{0}:=\left(\begin{array}{l}
u_{0} \\
u_{1}
\end{array}\right) \in D(A) .
\end{aligned}
$$

Teorema 3.1 El Operador A definido arriba genera $S(t)$ un semigrupo de contracción en un espacio de Hilbert $H$.

Prueba.- Observemos que $D(A)$ es denso en $H$. Probaremos que $A$ es disipativo.

Sea $U=(u, v)^{T} \in D(A)$ entonces

$$
\begin{aligned}
\langle A U, U\rangle & =\sum_{i=1}^{N} \int_{\Omega} \frac{\partial v}{\partial x_{i}} \frac{\overline{\partial u}}{\partial x_{i}} d x+\int_{\Omega}(\Delta u-a(x) v) \bar{v} d x \\
& =\sum_{i=1}^{N} \int_{\Omega} \frac{\partial v}{\partial x_{i}} \frac{\overline{\partial u}}{\partial x_{i}} d x+\int_{\Omega} \Delta u \bar{v}-a(x)|v|^{2} d x \\
& =\sum_{i=1}^{N} \int_{\Omega} \frac{\partial v}{\partial x_{i}} \frac{\overline{\partial u}}{\partial x_{i}}-\frac{\partial v}{\partial x_{i}} \frac{\overline{\partial v}}{\partial x_{i}} d x-\int_{\Omega} a(x)|v|^{2} d x \\
& =\sum_{i=1}^{N} \int_{\Omega} 2 i \operatorname{Im}\left(\frac{\partial u}{\partial x_{i}} \frac{\overline{\partial v}}{\partial x_{i}}\right) d x-\int_{\Omega} a(x)|v|^{2} d x
\end{aligned}
$$

donde $\operatorname{Im}(z)$ es la parte imaginaria de $z \in \mathbb{C}$. Tomando la parte reald e la igualdad (3.2), tenemos

$$
\operatorname{Re}\langle A U, U\rangle_{H_{0}^{1}(\Omega) \times L^{2}(\Omega)}=-\int_{\Omega} a(x)|v|^{2} d x \leq 0
$$

Ahora, probaremos que $0 \in \rho(A)$. En efecto, sea $F=(f, g)^{T} \in H_{0}^{1}(\Omega) \times L^{2}(\Omega)=H$, probaremos que existe $U=(u, v)^{T} \in D(A)$, tal que $A U=F$. Consideremos las ecuaciones

$$
\begin{gathered}
v=f \in H_{0}^{1}(\Omega) \\
\Delta u-a(x) v=g \in L^{2}(\Omega) .
\end{gathered}
$$


Reemplazando (3.3) en (3.4), tenemos

$$
\Delta u=a(x) f+g \in L^{2}(\Omega) .
$$

Por resultado estandar en ecuaciones lineales elípticas, tenemos que (3.5) tiene únicamente una solución $u \in H^{2}(\Omega) \cap H_{0}^{1}(\Omega)$. De (3.3) obtenemos $v=f$. Esto es, $A$ es una aplicación sobreyectiva. Afirmamos que $A$ es una aplicación inyectiva. En efecto, sea $A U=0$ entonces

$$
\begin{gathered}
v=0 \\
\Delta u-a(x) v=0 .
\end{gathered}
$$

Reemplazando (3.6) en (3.7) tenemos $\Delta u=0$ y usando la identidad de Green tenemos

$$
|u|_{H_{0}^{1}(\Omega)}^{2}=\int_{\Omega}|\nabla u|^{2} d x=\int_{\Omega} \Delta u \bar{u} d x=0
$$

de aquí $u=0$ in $H_{0}^{1}(\Omega)$. De (3.6) tenemos que $v=0$. Por lo tanto $U=0$. i.e. $A$ es inyectiva.

Así, existe $A^{-1}: H \rightarrow D(A)$ pues $A$ es uno a uno y $H$ es la imagen de $A$.

Ahora, probaremos que $A^{-1}$ es acotado.

Multiplicando la ecuación (3.5) por $\bar{u}$ e integrando sobre $\Omega$, tenemos

$$
\int_{\Omega} \Delta u \bar{u} d x=\int_{\Omega}(a f+g) \bar{u} d x
$$

pero desde que $\int_{\Omega}|\nabla u|^{2} d x=\int_{\Omega} \Delta u \bar{u} d x$, usando las desigualdades de Holder y Poincaré, obtenemos

$$
\begin{aligned}
\int_{\Omega}|\nabla u|^{2} d x & =\int_{\Omega}(a f+g) \bar{u} d x \\
& \leq|u|_{L^{2}}|a f+g|_{L^{2}} \\
& \leq \varepsilon \int_{\Omega}|u|^{2} d x+C(\varepsilon) \int_{\Omega}|a f+g|^{2} d x
\end{aligned}
$$

Entonces, tomando $\varepsilon>0$ tal que $1-\varepsilon C_{p}>0$ tenemos

$$
\left(1-\varepsilon C_{p}\right) \int_{\Omega}|\nabla u|^{2} d x \leq C(\varepsilon) \int_{\Omega}|a f+g|^{2} d x,
$$

esto es

$$
\begin{aligned}
\sqrt{1-\varepsilon C_{p}}|\nabla u|_{L^{2}} & \leq \sqrt{C(\varepsilon)}|a f+g|_{L^{2}} \\
& \leq \frac{\sqrt{C(\varepsilon)}}{\sqrt{1-\varepsilon C_{p}}}\left\{|a|_{\infty}|f|_{L^{2}}+|g|_{L^{2}}\right\}
\end{aligned}
$$


Así, usando (3.8), $v=f$, y las desigualdades de Holder y Poincaré conseguimos

$$
\begin{aligned}
|U|_{H} & =|\nabla u|_{L^{2}}+|v|_{L^{2}} \\
& =|\nabla u|_{L^{2}}+|f|_{L^{2}} \\
& =\hat{C}\left\{|f|_{L^{2}}+|g|_{L^{2}}\right\} \\
& =\hat{C}\left\{|\nabla f|_{L^{2}}+|g|_{L^{2}}\right\} .
\end{aligned}
$$

Entonces,

$$
|U|_{H} \leq \hat{C}|A U|_{H}
$$

esto es

$$
\left|A^{-1} F\right|_{H} \leq \hat{C}|F|_{H}
$$

lo cual nos permite decir que $A^{-1}$ es acotado.

Por el Teorema de Lummer Phillips, tenemos que $A$ es el generador infinitesimal de un $C_{0}$ semigrupo de contracción en $H: S(t)$.

Observación 3.1 Por el Teorema 4.3.2 en [4], si $D(A) \ni U$ entonces $S(t) U \in C^{1}([0, \infty), H) \cap C([0, \infty), D(A))$.

Observación 3.2 Por la observación 4.3.3 en [4] entonces $U(t):=S(t) U_{0}$ es la solución del PVI (3.1) y es única.

De las dos observaciones, conseguimos el siguiente resultado.

Proposición 3.1 Existe únicamente una solución de (3.1),

$$
U(t) \in C^{1}\left([0, \infty), H_{0}^{1}(\Omega) \times L^{2}(\Omega)\right) \cap C\left([0, \infty),\left(H^{2}(\Omega) \cap H_{0}^{1}(\Omega)\right) .\right.
$$

\section{Ahora, culminaremos la prueba del Teorema 2.1}

Desde que $U_{0}=\left(u^{0}, u^{1}\right) \in D(A)$ por proposición 3.1 , obtenemos que existe

$U(t) \in C^{1}\left([0, \infty), H_{0}^{1}(\Omega) \times L^{2}(\Omega)\right) \cap C\left([0, \infty),\left(H^{2}(\Omega) \cap H_{0}^{1}(\Omega)\right) \times H_{0}^{1}(\Omega)\right)$ solución de (3.1) tal que $U(0)=U_{0}, U(t) \in D(A), \forall t \in \mathbb{R}^{+}$.

Desde que $U$ satisface (3.1) tenemos $u_{t}=v$ y $v_{t}=\Delta u-a v$.

Por otro lado, tenemos $u_{t} \in C^{0}\left(\mathbb{R}^{+}, H_{0}^{1}(\Omega)\right)$, pero desde que $u \in C^{0}\left(\mathbb{R}^{+}, H_{0}^{1}(\Omega)\right)$ entonces $u \in C^{1}\left(\mathbb{R}^{+}, H_{0}^{1}(\Omega)\right)$.

Por otro lado, $u_{t t}=v_{t}=\Delta u-a u_{1} \in C\left(\mathbb{R}^{+}, L^{2}(\Omega)\right)$, pero $u_{t}$ y $u$ pertenecen a $C\left(\mathbb{R}^{+}, L^{2}(\Omega)\right)$ entonces $u \in C^{2}\left(\mathbb{R}^{+}, L^{2}(\Omega)\right)$. También obtenemos que $u \in C\left(\mathbb{R}^{+}, H^{2}(\Omega) \cap H_{0}^{1}(\Omega)\right)$. 
Observación 3.3 Por el Teorema de Hille Yosida (Teorema 4.4.3 en [4]), desde que A es el generador infinitesimal de un semigrupo de contracción, entonces $A$ es cerrado, $D(A)$ es denso en $H$ y $\forall \lambda>0, \exists(\lambda I-A)^{-1}$ acotado, más aún $\left\|(\lambda I-A)^{-1}\right\| \leq \frac{1}{\lambda}$.

Observación 3.4 Desde $A$ es cerrado y existe $A^{-1}$ (probado en $0 \in \rho(A)$ ), entonces $A^{-1}$ es también cerrado.

\section{USANDO EL MÉTODO DEL MULTIPLICADOR}

Sea $\left(u^{0}, u^{1}\right) \in H^{m+1}(\Omega) \times H^{m}(\Omega)$ satisfaciendo la condición de compatibalidad de m-ésimo orden. Entonces, la regularidad dada por (2.3) justifica los cálculos que haremos.

Sabemos que el problema (1.1) - (1.3) es disipativo.

\section{Lema 4.1}

$$
E^{\prime}(t)=-\int_{\Omega} a(x)\left|u_{t}\right|^{2} d x, \forall t>0
$$

Prueba.- Multiplicando la ecuación (1.1) por $u_{t}$ e integrando sobre $\Omega$, y usando la identidad de Green, tenemos

$$
\begin{aligned}
0 & =\int_{\Omega}\left(u_{t t}-\Delta u+a(x) u_{t}\right) u_{t} d x \\
& =\frac{\partial}{\partial t}\left\{\frac{1}{2} \int_{\Omega}\left(u_{t}\right)^{2} d x\right\}-\int_{\Omega}(\Delta u) u_{t} d x+\int_{\Omega} a(x)\left(u_{t}\right)^{2} d x \\
& =\frac{\partial}{\partial t}\left\{\frac{1}{2} \int_{\Omega}\left(u_{t}\right)^{2} d x\right\}-\int_{\Omega}(\nabla u) \nabla u_{t} d x+\int_{\Omega} a(x)\left(u_{t}\right)^{2} d x \\
& =\frac{\partial}{\partial t}\left\{\frac{1}{2} \int_{\Omega}\left(u_{t}\right)^{2} d x\right\}+\frac{\partial}{\partial t}\left\{\frac{1}{2} \int_{\Omega}|\nabla u|^{2} d x\right\}+\int_{\Omega} a(x)\left(u_{t}\right)^{2} d x,
\end{aligned}
$$

luego se obtiene el resultado.

Sea $\sigma \geq 0$, y $\phi: \mathbb{R}^{+} \rightarrow \mathbb{R}^{+}$una función $C^{2}$ cóncova y creciente. Sea $w$ una vecindad de la frontera $\partial \Omega$.

Lema 4.2 Sea $h: \bar{\Omega} \rightarrow \mathbb{R}^{N}$ un campo vector $C^{1}, \sigma \geq 0$ y $0 \leq S \leq T<+\infty$. Entonces tenemos,

$$
\begin{aligned}
& \int_{S}^{T} E^{\sigma} \phi^{\prime} \int_{\partial \Omega} 2 \partial_{v} u h \cdot \nabla u+(h \cdot v)\left(\left|u_{t}\right|^{2}-|\nabla u|^{2}\right) \\
& =\left[E^{\sigma} \phi^{\prime} \int_{\Omega} q u_{t} h \cdot \nabla u\right]_{S}^{T}-\int_{S}^{T}\left(\sigma E^{\prime} E^{\sigma-1} \phi^{\prime}+E^{\sigma} \phi^{\prime \prime}\right) \int_{\Omega} 2 u_{t} h \cdot \nabla u \\
& +\int_{S}^{T} E^{\sigma} \phi^{\prime} \int_{\Omega}(\operatorname{div} h)\left(\left|u_{t}\right|^{2}-|\nabla u|^{2}\right)+2 \sum_{i j} \frac{\partial h_{k}}{\partial x_{i}} \frac{\partial u}{\partial x_{i}} \frac{\partial u}{\partial x_{k}}+2 a u_{t} h \cdot \nabla u .
\end{aligned}
$$


Prueba.- Multiplicando la ecuación (1.1) por $E^{\sigma} \phi^{\prime} 2 h . \nabla u$ e integrando sobre $[S, T] \times \Omega$ tenemos

$$
\begin{aligned}
0= & \int_{S}^{T} E^{\sigma} \phi^{\prime} \int_{\Omega} 2 h \cdot \nabla u\left(u_{t t}-\Delta u+a(x) u_{t}\right) d x d t \\
= & \underbrace{\int_{S}^{T} E^{\sigma} \phi^{\prime} \int_{\Omega} 2 h \cdot(\nabla u) u_{t t} d x d t}_{I_{1}:=}-\underbrace{\int_{S}^{T} E^{\sigma} \phi^{\prime} \int_{\Omega} 2 h \cdot \nabla u(\Delta u) d x d t+}_{I_{2}:=} \\
& \int_{S}^{T} E^{\sigma} \phi^{\prime} \int_{\Omega} 2 h \cdot \nabla u\left(a(x) u_{t}\right) d x . d t
\end{aligned}
$$

Desde que $\frac{\partial}{\partial t}\left(\int_{\Omega} 2 h(\nabla u) u_{t} d x\right)=\int_{\Omega} 2 h(\nabla u) u_{t} d x+\int_{\Omega} 2 h(\nabla u) u_{t t} d x$ e integrado por partes obtenemos

$$
\begin{aligned}
I_{1} & =\int_{S}^{T} E^{\sigma} \phi^{\prime} \frac{\partial}{\partial t}\left(\int_{\Omega} 2 h(\nabla u) u_{t} d x\right) d t-\int_{S}^{T} E^{\sigma} \phi^{\prime} \int_{\Omega} 2 h\left(\nabla u_{t}\right) u_{t} d x d t \\
& =\int_{S}^{T} E^{\sigma} \phi^{\prime} \int_{\Omega} 2 h\left(\nabla u_{t}\right) u_{t} d x d t+\left[E^{\sigma} \phi^{\prime} \int_{\Omega} 2 h(\nabla \cdot u) u_{t} d x\right]_{S}^{T} \\
& =-\int_{S}^{T} E^{\sigma} \phi^{\prime} \int_{\Omega} 2 h\left(\nabla u_{t}\right) u_{t} d x d t .
\end{aligned}
$$

Usando la Identidad de Green tenemos

$$
\begin{aligned}
I_{2} & =-\int_{S}^{T} E^{\sigma} \phi^{\prime} \int_{\Omega} 2 h \cdot \nabla u \Delta u d x d t \\
& =\int_{S}^{T} E^{\sigma} \phi^{\prime} \int_{\Omega} \nabla u \cdot \nabla(2 h \cdot \nabla u) d x d t-\int_{S}^{T} E^{\sigma} \phi^{\prime} \int_{\partial \Omega} \frac{\partial u}{\partial v}(2 h \cdot \nabla u) d t .
\end{aligned}
$$

Reemplazando $I_{2}$ y $I_{2}$ en la igualdad (4.3) obtenemos

$$
\begin{aligned}
0= & -\int_{S}^{T} E^{\sigma} \phi^{\prime} \int_{\Omega} 2 h(\nabla u) u_{t} d x d t+\left[E^{\sigma} \phi^{\prime} \int_{\Omega} 2 h(\nabla u) u_{t} d x\right]_{S}^{T} \\
& \underbrace{-\int_{S}^{T} E^{\sigma} \phi^{\prime} \int_{\Omega} 2 h\left(\nabla u_{t}\right) u_{t} d x d t}_{I_{3}:=}+\underbrace{\int_{S}^{T} E^{\sigma} \phi^{\prime} \int_{\Omega} \nabla u \cdot \nabla(2 h \cdot \nabla u) d x d t}_{I_{4}:=} \\
& -\int_{S}^{T} E^{\sigma} \phi^{\prime} \int_{\partial \Omega} \frac{\partial u}{\partial v}(2 h . \nabla u) d t+\int_{S}^{T} E^{\sigma} \phi^{\prime} \int_{\Omega} 2 h \cdot \nabla u\left(a(x) u_{t}\right) d x \cdot d t
\end{aligned}
$$

Usando el hecho que $h \nabla\left(u_{t}^{2}\right)=\operatorname{div}\left(h u_{t}^{2}\right)-(\operatorname{div} h) u_{t}^{2}$ y el Teorema de la Divergencia $\int_{\partial \Omega} h u_{t}^{2} \cdot v=\int_{\Omega} \operatorname{div}\left(h u_{t}^{2}\right) d x$, tenemos 


$$
\begin{aligned}
I_{3} & =-\int_{S}^{T} E^{\sigma} \phi^{\prime} \int_{\Omega} h \cdot \nabla\left(u_{t}^{2}\right) d x d t \\
& =-\int_{S}^{T} E^{\sigma} \phi^{\prime} \int_{\Omega} \operatorname{div}\left(h u_{t}^{2}\right) d x d t+\int_{S}^{T} E^{\sigma} \phi^{\prime} \int_{\Omega}(\operatorname{div} h) u_{t}^{2} d x d t \\
& =-\int_{S}^{T} E^{\sigma} \phi^{\prime} \int_{\partial \Omega}\left(h u_{t}^{2}\right) \cdot v d x d t+\int_{S}^{T} E^{\sigma} \phi^{\prime} \int_{\Omega}(\operatorname{div} h) u_{t}^{2} d x d t .
\end{aligned}
$$

Por otro lado, tenemos

$$
\nabla u \cdot \nabla(2 h \cdot \nabla u)=2 \partial_{i} u \partial_{i} h_{k} \partial_{k} u+h \cdot \nabla\left(|\nabla u|^{2}\right)
$$

Y desde que $\operatorname{div}\left(h|\nabla u|^{2}\right)=(\operatorname{divh})|\nabla u|^{2}+h \nabla\left(|\nabla u|^{2}\right)$ y usando el Teorema de la Divergencia, conseguimos

$$
\int_{\Omega}(\operatorname{div} h)|\nabla u|^{2} d x+\int_{\Omega} h \nabla\left(|\nabla u|^{2}\right) d x=\int_{\Omega} \operatorname{div}\left(h|\nabla u|^{2}\right) d x=\int_{\partial \Omega} h|\nabla u|^{2} . v .
$$

Usando (4.5) y (4.6) obtenemos

$$
\begin{aligned}
I_{4} & =2 \int_{S}^{T} E^{\sigma} \phi^{\prime} \int_{\Omega} \partial_{i} u \partial_{i} h_{k} \partial_{k} u d x d t+\int_{S}^{T} E^{\sigma} \phi^{\prime} \int_{\Omega} h \cdot \nabla\left(|\nabla u|^{2}\right) d x d t \\
& =2 \int_{S}^{T} E^{\sigma} \phi^{\prime} \int_{\Omega} \partial_{i} u \partial_{i} h_{k} \partial_{k} u d x d t+\int_{S}^{T} E^{\sigma} \phi^{\prime} \int_{\partial \Omega} h|\nabla u|^{2} \cdot v d t \\
& =-\int_{S}^{T} E^{\sigma} \phi^{\prime} \int_{\Omega}(\operatorname{div} h)|\nabla u|^{2} d x d t .
\end{aligned}
$$

Reemplazando $I_{3}$ y $I_{4}$ en la igualdad (4.4), tenemos el resultado.

Lema 4.3 Existe una constante $C>0$ tal que $\forall 0 \leq S<T<\infty$ se verifica:

$$
\int_{S}^{T} E(t)^{1+\sigma} \phi^{\prime}(t) d t \leq C E(S)^{1+\sigma}+C \int_{S}^{T} E(t)^{\sigma} \phi^{\prime}(t)\left(\int_{w}\left|u_{t}\right|^{2} d x\right) d t
$$

Prueba.- Sea $K_{1}$ un conjunto compacto de $\Omega$ tal que $\Omega-K_{1}$ sea un compacto en $w$.

Defina $h(x):=\beta(x) m(x)$, donde $m(x)=x-x_{0}$ y $\beta$ es una función $C^{\infty}$ cuyo soporte está compactamente en $\Omega$ y es igual a 1 en $K_{1}$. Desde que $\phi^{\prime}$ es no creciente y positiva entonces $\phi^{\prime}$ está acotado en $\mathbb{R}^{+}$(i.e. $\left.\left|\phi^{\prime}(t)\right| \leq M\right)$.

Ahora, aplicamos (4.2) a esta $h$ y conseguimos

$$
\begin{aligned}
0 \geq & {\left[E^{\sigma} \phi^{\prime} \int_{\Omega} 2 u_{t} h \cdot \nabla u\right]_{S}^{T}-\int_{S}^{T}\left(\sigma E^{\prime} E^{\sigma-1} \phi^{\prime}+E^{\sigma} \phi^{\prime \prime}\right) \int_{\Omega} 2 u_{t} h . \nabla u } \\
& +\int_{S}^{T} E^{\sigma} \phi^{\prime} \int_{\Omega} \operatorname{div} h\left(u_{t}^{2}-|\nabla u|^{2}\right)+2 \sum_{i j} \frac{\partial h_{k}}{\partial x_{i}} \frac{\partial u}{\partial x_{i}} \frac{\partial u}{\partial x_{k}}+2 a(x) u_{t} h . \nabla u .
\end{aligned}
$$


Por otro lado, usando que $\int_{\Omega} 2 h . \nabla u u_{t} d x \leq c E(t)$ y $E(T)^{\sigma+1}<E(S)^{\sigma+1}$ para $S<T$, tenemos

$$
\begin{aligned}
& \left|\int_{S}^{T}\left(\sigma E^{\prime} E^{\sigma-1} \phi^{\prime}+E^{\sigma} \phi^{\prime \prime}\right)\left(\int_{\Omega} 2 h \cdot \nabla u u_{t} d x\right) d x\right| \\
& \leq \int_{S}^{T}\left|\left(\sigma E^{\prime} E^{\sigma-1} \phi^{\prime}+E^{\sigma} \phi^{\prime \prime}\right)\left(\int_{\Omega} 2 h \cdot \nabla u u_{t} d x\right) d x\right| \\
& \leq \int_{S}^{T}\left|\sigma E^{\prime} E^{\sigma-1} \phi^{\prime}+E^{\sigma} \phi^{\prime \prime}\right| c E d t \\
& =\int_{S}^{T}\left\{-\sigma E^{\prime} E^{\sigma-1} \phi^{\prime}-E^{\sigma} \phi^{\prime \prime}\right\} c E d t \\
& \leq c M \int_{S}^{T}-\sigma E^{\prime} E^{\sigma} d t+c E(S)^{\sigma+1} \int_{S}^{T}-\phi^{\prime \prime} d t \\
& =c M\left[\frac{\sigma}{\sigma+1} E(t)^{\sigma+1}\right]_{T}^{S}+c E(S)^{\sigma+1} \underbrace{\left[\phi^{\prime}\right]_{T}^{S}}_{\leq 2 M} \\
& \leq c M \frac{\sigma}{\sigma+1} E(S)^{\sigma+1}+c 2 M E(S)^{\sigma+1} . \\
& \leq c^{\prime} E(S)^{\sigma+1} .
\end{aligned}
$$

Y, desde que $E(T)<E(S)$ y por la desigualdad de Holder $\int_{\Omega} u_{t} h . \nabla u d x \leq\left\|u_{t}\right\|\|\nabla u\| \leq E(t)$, obtenemos

$$
\begin{aligned}
-\left[E^{\sigma} \phi^{\prime} \int_{\Omega} 2 u_{t} h \cdot \nabla u\right]_{S}^{T} & \leq E^{\sigma}(T) \phi^{\prime}(T) \int_{\Omega} 2 u_{t}(T) h(T) \cdot \nabla u(T) d x \\
& +E^{\sigma}(S) \phi^{\prime}(S) \int_{\Omega} 2 u_{t}(S) h(S) \cdot \nabla u(S) d x \\
& \leq E^{\sigma}(S) C\{E(S)+E(T)\} \leq C E^{\sigma+1}(S) .
\end{aligned}
$$

Aquí necesitamos hacer la siguiente estimativa

$$
\begin{aligned}
-\int_{S}^{T} E^{\sigma} \phi^{\prime} \int_{\Omega} 2 \sum_{i j} \frac{\partial h_{k}}{\partial x_{i}} \frac{\partial u}{\partial x_{i}} \frac{\partial u}{\partial x_{k}} d x d t & \leq \int_{S}^{T} E^{\sigma} \phi^{\prime} c\|\nabla u\|\|\nabla u\|_{L^{2}\left(\Omega-K_{1}\right)} d t \\
& \leq \int_{S}^{T} E^{\sigma} \phi^{\prime}\left\{\varepsilon E(t)+C(\varepsilon)\|\nabla u\|_{L^{2}\left(\Omega-K_{1}\right)}^{2}\right\} d t
\end{aligned}
$$

donde $\varepsilon$ será considerado lo suficientemente pequeño.

Usando las desigualdades (4.9), (4.10) y (4.11) EN (4.8) tenemos que existe $C>0$ tal que 


$$
\begin{aligned}
& \int_{S}^{T} E^{\sigma} \phi^{\prime} \int_{\Omega} N u_{t}^{2}+(2-N)|\nabla u|^{2} d x d t \\
& \leq C \int_{S}^{T} E^{\sigma} \phi^{\prime} \int_{\Omega-K_{1}}\left\{u_{t}^{2}+|\nabla u|^{2}\right\} d x d t+C E(S)^{1+\sigma}+\varepsilon \int_{S}^{T} E^{1+\sigma} \phi^{\prime} d t,
\end{aligned}
$$

$\operatorname{con} \varepsilon$ suficientemente pequeño.

\section{Integrando por partes la expresión:}

$$
0=(N-1) \int_{S}^{T} E^{\sigma} \phi^{\prime} \int_{\Omega} u\left(u_{t t}-\Delta u+a(x) u_{t} d x d t\right.
$$

tenemos

$$
\begin{aligned}
&(N-1) \int_{S}^{T} E^{\sigma} \phi^{\prime} \int_{\Omega}|\nabla u|^{2} d x d t-(N-1) \int_{S}^{T} E^{\sigma} \phi^{\prime} \int_{\Omega}\left|u_{t}\right|^{2} d x d t \\
&=-(N-1)\left[E^{\sigma} \phi^{\prime} \int_{\Omega} u u_{t} d x\right]_{S}^{T} \\
& \quad+(N-1) \int_{S}^{T}\left(\sigma E^{\sigma-1} E^{\prime} \phi^{\prime}+E^{\sigma} \phi^{\prime \prime}\right) \int_{\Omega} u u_{t} d x d t \\
&-(N-1) \int_{S}^{T} E^{\sigma} \phi^{\prime} \int_{\Omega} u a u_{t} d x d t .
\end{aligned}
$$

Por la desigualdad de Poincaré tenemos $\int_{\Omega} u u_{t} d x \leq\|u\|\left\|u_{t}\right\| \leq C_{p}\|\nabla u\|\left\|u_{t}\right\| \leq C E(t)$. Usando esto en (4.9) obtenemos

$$
\left|(N-1) \int_{S}^{T}\left(\sigma E^{\sigma-1} E^{\prime} \phi^{\prime}+E^{\sigma} \phi^{\prime \prime}\right) \int_{\Omega} u u_{t} d x d t\right| \leq C E(S)^{\sigma+1}
$$

Con una prueba similar a (4.10),también obtenemos

$$
-(N-1)\left[E^{\sigma} \phi^{\prime} \int_{\Omega} u u_{t} d x\right]_{S}^{T} \leq C E(S)^{\sigma+1}
$$

Sumando (4.12) y (4.13), tomando $\varepsilon<1$ usando (4.14) y (4.15) tenemos

$$
\begin{aligned}
\int_{S}^{T} E^{1+\sigma} \phi^{\prime} & \leq \int_{S}^{T} E^{\sigma} \phi^{\prime} \int_{\Omega} u_{t}^{2}+|\nabla u|^{2} d x d t \\
& \leq C E(S)^{1+\Omega}+C \int_{S}^{T} E^{\sigma} \phi^{\prime} \int_{\Omega-K_{1}}\left\{u_{t}^{2}+|\nabla u|^{2}\right\} d x d t
\end{aligned}
$$

Queremos eliminar el último término de (4.16). Para hacer esto, construimos una función $\xi \in C^{\infty}(\bar{\Omega})$ tal que $\xi=1$ en $\Omega-K_{1}$ y $\xi=0$ fuera de $w$. 
Multiplicamos la ecuación (1.1) por $\xi u$ e integramos sobre $\Omega$; luego multiplicamos esta expresión por $E^{\sigma} \phi^{\prime}$, e integramos sobre $[S, T]$, e integrando por partes conseguimos

$$
\begin{aligned}
& \int_{S}^{T} E^{\sigma} \phi^{\prime} \int_{\Omega}-\xi u a(x) u_{t} d x d t \\
& =\int_{S}^{T} E^{\sigma} \phi^{\prime} \int_{\Omega} \xi u\left(u_{t t}-\Delta u\right) d x d t \\
& =\int_{S}^{T} E^{\sigma} \phi^{\prime} \int_{\Omega} \xi u u_{t t} d x d t-\int_{S}^{T} E^{\sigma} \phi^{\prime} \int_{\Omega} \xi u \Delta u d x d t \\
& =-\int_{S}^{T}\left(\sigma E^{\sigma-1} E^{\sigma} \phi^{\prime}+E^{\sigma} \phi^{\prime \prime}\right) \int_{\Omega} \xi u u_{t t} d x d t \\
& =-\int_{S}^{T} E^{\sigma} \phi^{\prime} \int_{\Omega} \xi u_{t}^{2} d x d t+\left[E^{\sigma} \phi^{\prime} \int_{\Omega} u u_{t} d x\right]_{S}^{T} \\
& -\underbrace{\int_{S}^{T} E^{\sigma} \phi^{\prime} \int_{\Omega} \xi u \Delta u d x d t}_{I:=} .
\end{aligned}
$$

Usando la Identidad de Green y $\nabla\left(u^{2}\right)=(\nabla u) u+u \nabla u$ tenemos

$$
\begin{aligned}
I & =\int_{S}^{T} E^{\sigma} \phi^{\prime} \int_{\Omega} \nabla(\xi u) \cdot \nabla u d x d t \\
& =\int_{S}^{T} E^{\sigma} \phi^{\prime} \int_{\Omega}\{\nabla(\xi) u \cdot \nabla u+\xi \nabla u \cdot \nabla u\} d x d t \\
& =\int_{S}^{T} E^{\sigma} \phi^{\prime} \int_{\Omega}\left\{\frac{1}{2} \nabla(\xi) \cdot \nabla\left(u^{2}\right)+\xi|\nabla u|^{2}\right\} d x d t \\
& =\int_{S}^{T} E^{\sigma} \phi^{\prime} \int_{\Omega}\left\{\frac{1}{2}(\Delta \xi) u^{2}+\xi|\nabla u|^{2}\right\} d x \cdot d t
\end{aligned}
$$

Reemplazando (4.18) en (4.17) obtenemos

$$
\begin{aligned}
\int_{S}^{T} E^{\sigma} \phi^{\prime} \int_{\Omega}-\xi u a(x) u_{t} d x d t & =-\int_{S}^{T}\left(\sigma E^{\sigma-1} E^{\prime} \phi+E^{\sigma} \phi^{\prime \prime}\right) \int_{\Omega} \xi u u_{t} d x d t \\
& \left.+\int_{S}^{T} E^{\sigma} \phi^{\prime} \int_{\Omega} \xi|\nabla u|^{2} d x d t+E^{\sigma} \phi \int_{\Omega} u u_{t} d x\right]_{S}^{T} \\
& =\int_{S}^{T} E^{\sigma} \phi^{\prime} \int_{\Omega}\left\{\frac{1}{2}(\Delta \xi) u^{2}+\xi u_{t}^{2}\right\} d x d t
\end{aligned}
$$

de donde 


$$
\begin{aligned}
\int_{S}^{T} E^{\sigma} \phi^{\prime} \int_{\Omega-K_{1}} 1 \cdot|\nabla u|^{2} d x d t & \leq \int_{S}^{T} E^{\sigma} \phi^{\prime} \int_{\Omega} \xi|\nabla u|^{2} d x d t \\
& =\int_{S}^{T}\left(\sigma E^{\sigma-1} E^{\prime} \phi^{\prime}+E^{\sigma} \phi^{\prime \prime}\right) \int_{\Omega} \xi u u_{t} d x d t \\
& +\int_{S}^{T} E^{\sigma} \phi^{\prime} \int_{\Omega}-\xi u a(x) u_{t} d x d t \\
& +\int_{S}^{T} E^{\sigma} \phi^{\prime} \int_{\Omega}\left\{\frac{1}{2}(\Delta \xi) u^{2}+\xi u_{t}^{2}\right\} d x d t .
\end{aligned}
$$

Desde que $\xi$ es acotada, en forma similar a (4.9), obtenemos

$$
\left|\int_{S}^{T}\left(\sigma E^{\sigma-1} E^{\prime} \phi^{\prime}+E^{\sigma} \phi^{\prime \prime}\right) \int_{\Omega} \xi u u_{t} d x d t\right| \leq C E^{1+\sigma} .
$$

También, usando el hecho que $\xi$ es acotada, similarmente a (4.10) conseguimos

$$
-\left[E^{\sigma} \phi^{\prime} \int_{\Omega} u u_{t} d x\right]_{S}^{T} \leq C E^{1+\sigma} .
$$

Y, usando (4.21) y (4.22) en (4.20) obtenemos

$$
\int_{S}^{T} E^{\sigma} \phi^{\prime} \int_{\Omega-K_{1}}|\nabla u|^{2} d x d t \leq C E(S)^{1+\sigma}+C \int_{S}^{T} E^{\sigma} \phi^{\prime} \int_{w}\left(u_{t}^{2}+u^{2}\right) d x d t .
$$

Ahora, para alimentar el último término de (4.23), adaptaremos el método de Conrad y Rao [1].

Sea $\beta \in C^{\infty}\left(\mathbb{R}^{N}\right)$ tal que $0 \leq \beta \leq 1, \beta=1$ en $w$ y $\beta=0$ fuera de una vecindad de $w$.

Fijamos $t$ y consideramos $z$ la solución del problema elíptico:

$$
\begin{aligned}
\Delta z & =\beta(x) u \text { en } \Omega \\
\left.z\right|_{\partial \Omega} & =0
\end{aligned}
$$

Multiplicando la ecuación (4.24) por z, ingrando sobre $\Omega$ y usando la Identidad de Green, tenemos:

$$
\int_{\Omega} \beta(x) u z d x=\int_{\Omega}(\Delta z) z d x=-\int_{\Omega}|\nabla z|^{2} d x
$$

de aquí, usando las desigualdades de Holder y Poincaré, obtenemos

$$
|z|_{L^{2}}^{2} \leq C \int_{\Omega}|\nabla z|^{2} d x=-C \int_{\Omega} \beta(x) u z d x \leq c|u|_{L^{2}(\Omega)}|z|_{L^{2}(\Omega)}
$$


luego,

$$
|z|_{L^{2}} \leq C|u|_{L^{2}(\Omega)}
$$

Análogamente a (4.26) obtenemos

$$
\begin{aligned}
|z|_{L^{2}}^{2} \leq C \int_{\Omega}|\nabla z|^{2} d x & =-C \int_{\Omega} \beta(x) u z d x \\
& \leq c|\beta u|_{L^{2}(\Omega)}|z|_{L^{2}(\Omega)} \\
& \leq c|u|_{L^{2}(w)}|z|_{L^{2}(\Omega)},
\end{aligned}
$$

luego,

$$
|z|_{L^{2}} \leq C|u|_{L^{2}(w)}
$$

Diferenciando con respecto a $t$ a la ecuación (4.24) tenemos el problema

$$
\begin{aligned}
\Delta z_{t} & =\beta(x) u_{t} \text { en } \Omega \\
\left.z_{t}\right|_{\partial \Omega} & =0 .
\end{aligned}
$$

Multiplicando (4.30) por $z_{t}$, integrando sobre $\Omega$ y usando la Identidad de Green obtenemos

$$
\int_{\Omega} \beta(x) u_{t} z_{t} d x=\int_{\Omega}\left(\Delta z_{t}\right) z_{t} d x=-\int_{\Omega}\left|\nabla z_{t}\right|^{2} d x
$$

de aquí, usando las desigualdades de Holder y Poincaré, obtenemos

$$
\left|z_{t}\right|_{L^{2}}^{2} \leq C \int_{\Omega}\left|\nabla z_{t}\right|^{2} d x=-C \int_{\Omega} \beta(x) u_{t} z_{t} d x \leq c\left|u_{t}\right|_{L^{2}(\Omega)}\left|z_{t}\right|_{L^{2}(\Omega)}
$$

luego,

$$
\left|z_{t}\right|_{L^{2}} \leq C\left|u_{t}\right|_{L^{2}(\Omega)}
$$

También

$$
\begin{aligned}
\left|z_{t}\right|_{L^{2}}^{2} \leq C \int_{\Omega}\left|\nabla z_{t}\right|^{2} d x & =-C \int_{\Omega} \beta(x) u_{t} z_{t} d x \\
& \leq c\left|\beta u_{t}\right|_{L^{2}(\Omega)}\left|z_{t}\right|_{L^{2}(\Omega)} \\
& \leq c\left|u_{t}\right|_{L^{2}(w)}\left|z_{t}\right|_{L^{2}(\Omega)}
\end{aligned}
$$

luego

$$
\left|z_{t}\right|_{L^{2}} \leq C\left|u_{t}\right|_{L^{2}(w)}
$$


Por otro lado,

$$
\begin{aligned}
0 & =\int_{S}^{T} E^{\sigma} \phi^{\prime} \int_{\Omega} z\left(u_{t t}-\Delta u+a u_{t}\right) d x d t \\
& =\left[E^{\sigma} \phi^{\prime} \int_{\Omega} z u_{t} d x\right]_{S}^{T}-\int_{S}^{T}\left(\sigma E^{\prime} E^{\sigma-1} \phi^{\prime}+E^{\sigma} \phi^{\prime \prime}\right) \int_{\Omega} z u_{t} d x d t \\
& +\int_{S}^{T} E^{\sigma} \phi^{\prime} \int_{\Omega}\left(-z \Delta u+a z u_{t}-z_{t} u_{t}\right) d x d t
\end{aligned}
$$

Usando la Identidad de Green y el hecho que $z$ es solución de (4.24) - (4.25) obtenemos

$$
\begin{aligned}
\int_{S}^{T} E^{\sigma} \phi^{\prime} \int_{\Omega} z \Delta u d x d t & =\int_{S}^{T} E^{\sigma} \phi^{\prime} \int_{\Omega}(\Delta z) u d x d t \\
& =\int_{S}^{T} E^{\sigma} \phi^{\prime} \int_{\Omega}(\beta(x) u) u d x d t \\
& =\int_{S}^{T} E^{\sigma} \phi^{\prime} \int_{w} u^{2} d x d t
\end{aligned}
$$

Usando (4.37) en (4.36) tenemos

$$
\begin{aligned}
\int_{S}^{T} E^{\sigma} \phi^{\prime} \int_{w} u^{2} d x d t & =\int_{S}^{T} E^{\sigma} \phi^{\prime} \int_{\Omega} z \Delta u d x d t \\
& =\left[E^{\sigma} \phi^{\prime} \int_{\Omega} z u_{t} d x\right]_{S}^{T}-\int_{S}^{T}\left(\sigma E^{\prime} E^{\sigma-1} \phi^{\prime}+E^{\sigma} \phi^{\prime \prime}\right) \int_{\Omega} z u_{t} d x d t \\
& =\int_{S}^{T} E^{\sigma} \phi^{\prime} \int_{\Omega}\left(a z u_{t}-z_{t} u_{t}\right) d x d t
\end{aligned}
$$

Podemos observar que se verifica la siguiente desigualdad

$$
\left|\left[E^{\sigma} \phi^{\prime} \int_{\Omega} z u_{t} d x\right]_{S}^{T}-\int_{S}^{T}\left(\sigma E^{\prime} E^{\sigma-1} \phi^{\prime}+E^{\sigma} \phi^{\prime \prime}\right) \int_{\Omega} z u_{t} d x d t\right| \leq C E(S)^{1+\sigma} .
$$

Por otro lado, sea $\eta>0$, usando (4.35) obtenemos

$$
\begin{aligned}
\left|\int_{S}^{T} E^{\sigma} \phi^{\prime} \int_{\Omega} z_{t} u_{t} d x d t\right| & \leq \int_{S}^{T} E^{\sigma} \phi^{\prime} C\left|z_{t}\right|_{L^{2}}\left|u_{t}\right|_{L^{2}} d t \\
& \leq \int_{S}^{T} E^{\sigma} \phi^{\prime} C\left|u_{t}\right|_{L^{2}(w)}\left|u_{t}\right|_{L^{2}} d t \\
& \leq \frac{C}{2 \eta} \int_{S}^{T} E^{\sigma} \phi^{\prime} \int_{w} u_{t}^{2} d x d t+\frac{\eta}{2} \int_{S}^{T} E^{\sigma} \phi^{\prime} \int_{\Omega} u_{t}^{2} d x d t \\
& \leq \frac{C}{2 \eta} \int_{S}^{T} E^{\sigma} \phi^{\prime} \int_{w} u_{t}^{2} d x d t+\eta \int_{S}^{T} E^{\sigma+1} \phi^{\prime} d t
\end{aligned}
$$

donde $\eta$ será tomado lo suficientemente pequeño. 
También, tenemos

$$
\begin{aligned}
\left|\int_{S}^{T} E^{\sigma} \phi^{\prime} \int_{\Omega} z a u_{t} d x d t\right| & \leq \int_{S}^{T} E^{\sigma} \phi^{\prime}\left|\int_{\Omega} z a u_{t} d x\right| d t \\
& \leq \int_{S}^{T} E^{\sigma} \phi^{\prime}|z|_{L^{2}(\Omega)}\left|a u_{t}\right|_{L^{2}} d t \\
& \leq \int_{S}^{T} E^{\sigma} \phi^{\prime} C|u|_{L^{2}(w)}\left|\sqrt{a} u_{t}\right|_{L^{2}} d t \\
& \leq \gamma \int_{S}^{T} E^{\sigma} \phi^{\prime} \int_{w} u^{2} d x d t+C(\gamma) \int_{S}^{T} E^{\sigma} \phi^{\prime} \int_{\Omega} a u_{t}^{2} d x d t \\
& \leq \gamma \int_{S}^{T} E^{\sigma} \phi^{\prime} \int_{w} u^{2} d x d t+C(\gamma) \int_{S}^{T} E^{\sigma} \phi^{\prime}\left(-E^{\prime}\right) d t \\
& \leq \gamma \int_{S}^{T} E^{\sigma} \phi^{\prime} \int_{w} u^{2} d x d t-C(\gamma) \int_{S}^{T} E^{\sigma}\left(E^{\prime}\right) d t
\end{aligned}
$$

donde $\gamma$ será tomado muy pequeño. Desde que

$$
\begin{aligned}
-\int_{S}^{T} E^{\sigma} E^{\prime} d t & =-\frac{1}{\sigma+1}\left[E(t)^{\sigma+1}\right]_{S}^{T} \\
& =\frac{1}{\sigma+1}\left\{E(S)^{\sigma+1}-E(T)^{\sigma+1}\right\} \\
& =\frac{1}{\sigma+1}\left\{E(S)^{\sigma+1}\right\}
\end{aligned}
$$

entonces (4.41) queda expresada por

$$
\left|\int_{S}^{T} E^{\sigma} \phi^{\prime} \int_{\Omega} z a u_{t} d x d t\right| \leq \gamma \int_{S}^{T} E^{\sigma} \phi^{\prime} \int_{w} u^{2} d x d t+C(\gamma) E(S)^{\sigma+1}
$$

Usando (4.39), (4.40) y (4.42) en (4.38), tenemos

$$
\begin{aligned}
(1-\gamma) \int_{S}^{T} E^{\sigma} \phi^{\prime} \int_{w} u^{2} d x d t & \leq \frac{C}{\eta} \int_{S}^{T} E^{\sigma} \phi^{\prime} \int_{w} u_{t}^{2} d x d t \\
& +C E(S)^{1+\sigma}+\eta \int_{S}^{T} E^{1+\sigma} \phi^{\prime} d t
\end{aligned}
$$

Tomando $\gamma<1$, de (4.43) tenemos que existe $C>0$ tal que $\forall \eta>0$ se verifica

$$
\begin{aligned}
\int_{S}^{T} E^{\sigma} \phi^{\prime} \int_{w} u^{2} d x d t & \leq \frac{C}{\eta} \int_{S}^{T} E^{\sigma} \phi^{\prime} \int_{w} u_{t}^{2} d x d t \\
& +C E(S)^{1+\sigma}+\eta \int_{S}^{T} E^{1+\sigma} \phi^{\prime} d t
\end{aligned}
$$

donde $\eta$ es suficientemente pequeño. 
Reemplazando (4.44) en (4.23) conseguimos

$$
\begin{aligned}
& \int_{S}^{T} E^{\sigma} \phi^{\prime} \int_{\Omega-K_{1}}|\nabla u|^{2} d x d t \\
& \leq C E(S)^{1+\sigma}+\left(C+\frac{C}{\eta}\right) \int_{S}^{T} E^{\sigma} \phi^{\prime} \int_{w} u_{t}^{2} d x d t+\eta \int_{S}^{T} E^{1+\sigma_{\phi^{\prime}} d t}
\end{aligned}
$$

Reemplazando (4.45) en (4.16) tenemos

$$
(1-\eta) \int_{S}^{T} E^{1+\sigma} \phi^{\prime} d t \leq C E(S)^{1+\sigma}+\left(C+\frac{C}{\eta}\right) \int_{S}^{T} E^{\sigma} \phi^{\prime} \int_{w} u_{t}^{2} d x d t
$$

y tomando $\eta<1$ obtenemos

$$
\int_{S}^{T} E^{1+\sigma^{\prime}} d t \leq C E(S)^{1+\sigma}+C \int_{S}^{T} E^{\sigma} \phi^{\prime} \int_{w} u_{t}^{2} d x d t
$$

\section{CULMINANDO LA PRUEBA DEL TEOREMA PRINCIPAL}

Sea $\rho: t \rightarrow \rho(t)$ una función decreciente que va para cero cuando $t$ va para el infinito. Posteriormente, eligiremos $\rho$.

Definamos la función $\tilde{\alpha}$ por

$$
\begin{array}{lll}
\tilde{\alpha}(r, t):=\tilde{a}(r) & \text { si } & \frac{R}{2} \leq r \leq R-\rho(t) \\
\tilde{\alpha}(r, t):=\tilde{a}(R-\rho(t)) & \text { si } & r \leq R-\rho(t)
\end{array}
$$

y la función $\alpha$ en $w \times \mathbb{R}^{+}$por

$$
\alpha(x, t):=\tilde{\alpha}(|x|, t), \quad \forall|x| \geq \frac{R}{2} .
$$

Lema 5.1 (Gagliardo-Niremberg) Si $m>\frac{N}{2}$, existe $c>0$ tal que para cada $v \in H^{m}(\Omega)$ vale

$$
\|v\|_{L^{\infty}(\Omega)} \leq c\|v\|_{H^{m}(\Omega)}^{\theta}\|v\|_{L^{2}(\Omega)}^{1-\theta} \operatorname{con} \theta=\frac{N}{2 m}
$$

Ahora, usando (2.3) y (2.4) es posible aplica (5.3) y deducir

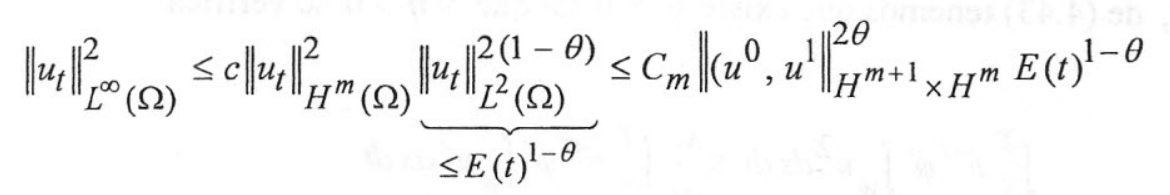

Sea $p>0$ tal que valga (2.9). Entonces, usando la desigualdad de Jensen y (5.4) estimaremos el último de (4.7). 


$$
\begin{aligned}
& \int_{S}^{T} E(t)^{\sigma} \phi^{\prime}(t)\left(\int_{w} u_{t}^{2} d x\right) d t \\
& =\int_{S}^{T} E(t)^{\sigma}(t)\left(\int_{w} \frac{1}{\alpha(x, t)} u_{t}^{2} \alpha(x, t) d x\right) d t \\
& \leq \int_{S}^{T} E(t)^{\sigma} \phi^{\prime}(t)\left\|u_{t}^{2} \alpha^{\frac{p}{p+1}}\right\|_{L} \frac{p+1}{p}\left\|\alpha^{-\frac{p}{p+1}}\right\|_{L^{p+1} d t} \\
& =\int_{S}^{T} E(t)^{\sigma}(t)\left(\int_{w} \frac{1}{\alpha(x, t)^{p}} d x\right)^{\frac{1}{p+1}}\left(\int_{w} u_{t}^{\frac{2(p+1)}{p}} \alpha(x, t) d x\right)^{\frac{p}{p+1}} d t \\
& =\int_{S}^{T} E(t)^{\sigma}(t)^{\frac{1}{(p+1)}}\left(\phi^{\prime}(t)^{p} \int_{w} \frac{1}{\alpha(x, t)^{p}} d x\right)^{\frac{1}{p+1}}\left(\int_{w} u_{t}+\frac{2}{p} \alpha(x, t) d x\right)^{\frac{p}{p+1}} d t \\
& \leq \int_{S}^{T} E(t)^{\sigma} \phi^{\prime}(t)^{\frac{1}{(p+1)}}\left(\phi^{\prime}(t)^{p} \int_{w} \frac{1}{\alpha(x, t)^{p}} d x\right)^{\frac{1}{p+1}} \cdot \\
& \left(\int_{w} u_{t}^{2} \alpha(x, t) d x\right)^{\frac{p}{p+1}}\left\|u_{t}(t)\right\|_{L^{\infty}(\Omega)}^{\frac{2}{p+1}} d t \\
& \leq C_{m} \int_{S}^{T} E(t)^{\sigma+\frac{(1-\theta)}{(p+1)}} \phi^{\prime}(t)^{\frac{1}{(p+1)}}\left(\phi^{\prime}(t)^{p} \int_{w} \frac{1}{\alpha(x, t)^{p}} d x\right)^{\frac{1}{p+1}} \\
& \left(\int_{w} u_{t}^{2} \alpha(x, t) d x\right)^{\frac{p}{p+1}} d t .
\end{aligned}
$$

Para simplificar notaciones, introducimos

$$
\varepsilon(t)=\phi^{\prime}(t)\left(\int_{w} \frac{1}{\alpha(x, t)^{p}} d x\right)^{\frac{1}{p}} .
$$

Sea $\varepsilon>0$. Aplicando la desigualdad de Young conseguimos la siguiente estimativa

$$
\begin{aligned}
& \int_{S}^{T} E(t)^{\sigma} \phi^{\prime}\left(\int_{w} u_{t}^{2} d x\right) d t \\
& \leq C_{m} \int_{S}^{T} E(t)^{\sigma+\frac{(1-\theta)}{(p+1)}} \phi^{\prime}(t)^{\frac{1}{(p+1)}} \varepsilon(t)^{\frac{p}{p+1}}\left(\int_{w} u_{t}^{2} \alpha(x, t) d x\right)^{\frac{p}{p+1}} d t \\
& \leq C_{m} \int_{S}^{T} \underbrace{E(t)^{\sigma+\frac{(1-\theta)}{(p+1)}} \phi^{\prime}(t)^{\frac{1}{(p+1)}}}_{\in L^{p+1}} \underbrace{\left(\varepsilon(t)\left(\int_{w} u_{t}^{2} \alpha(x, t) d x\right)^{\frac{p}{p+1}}\right.}_{\in L^{\frac{p+1}{p}}} d t \\
& \leq C_{m}\left(\int_{S}^{T} E(t)^{\sigma(p+1)+(1-\theta)} \phi^{\prime}(t) d t\right)^{\frac{p}{(p+1)}} \cdot\left(\int_{S}^{T} \varepsilon(t) \int_{w} u_{t}^{2} \alpha(x, t) d x d t\right)^{\frac{p}{p+1}} \\
& \leq C_{m} \frac{\varepsilon}{p+1} \int_{S}^{T} E(t)^{\sigma(p+1)+(1-\theta)} \phi^{\prime}(t) d t+C_{m} \frac{p}{(p+1) \varepsilon} \int_{S}^{T} \varepsilon(t) \int_{w} u_{t}^{2} \alpha(x, t) d x d t .
\end{aligned}
$$


$\sigma$ es definida tal que

$$
\sigma(p+1)+(1-\theta)=\sigma+1, \text { esto es } \sigma=\frac{\theta}{p}=\frac{N}{2 m p} .
$$

De (4.7) y (5.7) podemos deducir: si $\varepsilon$ es suficientemente pequeño, existe una constante positiva $C$ tal que

$$
\int_{S}^{T} E(t)^{1+\sigma} \phi^{\prime}(t) d t \leq C E(S)^{\sigma+1}+C \int_{S}^{T} \varepsilon(t) \int_{w} u_{t}^{2} \alpha(x, t) d x d t .
$$

Ahora, eligiendo cuidadosamente $\rho$ y $\phi$, estimaremos el último término de (5.9).

\section{La elección de la función $\rho$}

Asumamos que $\phi$ es una función $C^{2}$ cóncava y estrictamente creciente tal que

$$
\phi(t) \rightarrow+\infty \quad \text { y } \quad \phi^{\prime}(t) \rightarrow 0 \quad \text { cuando } t \rightarrow+\infty
$$

Lema 5.2 Si b satisface (2.9), entonces existe $C>0$ tal que

$$
\begin{aligned}
\int_{\frac{R}{2}}^{R} \frac{1}{\tilde{\alpha}(r, t)^{p}} d r & =\int_{\frac{R}{2}}^{R-\rho(t)} \frac{1}{\tilde{\alpha}(r, t)^{p}} d r+\int_{R-\rho(t)}^{R} \frac{1}{\tilde{\alpha}(r, t)^{p}} d r \\
& =\int_{\frac{R}{2}}^{R-\rho(t)} \frac{1}{\tilde{\alpha}(r)^{p}} d r+\int_{R-\rho(t)}^{R} \frac{1}{\tilde{\alpha}(R-\rho(t))^{p}} d r \\
& =\int_{\rho(t)}^{\frac{R}{2}} \frac{1}{b(r)^{p}} d r+\int_{R}^{R}-\rho(t) \frac{1}{b(\rho(t))^{p}} d r \\
& \leq C \frac{\rho(t)}{b(\rho(t))^{p}}+\frac{\rho(t)}{b(\rho(t))^{p}} .
\end{aligned}
$$

Usando (5.11) obtenemos la siguiente estimativa para $\varepsilon$.

$$
\varepsilon(t) \leq \phi^{\prime}(t) \frac{\rho(t)^{\frac{1}{p}}}{b(\rho(t))^{p}} .
$$

Desde que $b$ es estrictamente creciente próximo 0 , definimos $\rho$ :

$$
\rho(t):=b^{-1}\left(\phi^{\prime}(t)\right)
$$

Observamos que $\rho$ es decreciente, desde que $b$ es creciente y $\phi^{\prime}$ es decreciente. De la definición de $\rho$ y (5.12) tenemos

$$
\varepsilon(t) \leq C_{\rho}(t)^{\frac{1}{p}}
$$

También, obtenemos 


$$
\begin{aligned}
\int_{w} \alpha(x, t) u_{t}^{2} d x & =\int_{\frac{R}{2} \leq|x| \leq R-\rho(t)} \alpha(x, t) u_{t}^{2} d x+\int_{|x|>R-\rho(t)} \alpha(x, t) u_{t}^{2} d x \\
& \leq \int_{\Omega} a(x) u_{t}^{2} d x+\int_{|x|>R-\rho(t)} \tilde{a}(R-\rho(t)) u_{t}^{2} d x \\
& \leq-E^{\prime}(t)+b(\rho(t)) E(t) \\
& =-E^{\prime}(t)+\phi^{\prime}(t) E(t) .
\end{aligned}
$$

De (5.9), usando (5.15), (5.14), $\rho(t)^{\frac{1}{p}} \leq \rho(S)^{\frac{1}{p}}$ y el hecho que $E$ decrece, tenemos

$$
\begin{aligned}
& \int_{S}^{T} E(t)^{\sigma+1} \phi^{\prime}(t) d t \\
& \leq C E(S)^{1+\sigma}+C \int_{S}^{T} \varepsilon(t)\left(-E^{\prime}(t)+\phi^{\prime}(t) E(t)\right) d t \\
& \leq C E(S)^{1+\sigma}+C \int_{S}^{T} \rho(S)^{\frac{1}{p}}\left(-E^{\prime}(t)\right) d t+C \int_{S}^{T} \rho(t)^{\frac{1}{p}} \phi^{\prime}(t) E(S) d t \\
& \leq C E(S)^{1+\sigma}+C \rho(S)^{\frac{1}{p}}\{E(S)-E(T)\} C E(S) \int_{S}^{T} \rho(t)^{\frac{1}{p}} \phi^{\prime}(t) d t \\
& \leq C E(S)^{1+\sigma}+C \rho(S)^{\frac{1}{p}} E(S)+C E(S) \int_{S}^{T} \rho(t)^{\frac{1}{p}} \phi^{\prime}(t) d t .
\end{aligned}
$$

\section{La elección de la función $\phi$}

Aquí, mostraremos como definir $\phi$ de modo que $\int_{1}^{+\infty} \rho(t)^{\frac{1}{p}} \phi^{\prime}(t) d t$ sea finito.

Sea $p^{\prime}>1+p$ y defina

$$
\psi(t):=+\int_{1}^{t} \frac{1}{b\left(\frac{1}{r^{p^{\prime}}}\right)} d r, \quad \forall t>1 .
$$

Entonces, $\psi$ es una función $C^{2}$ convexa y estrictamente creciente, que satisface

$$
\psi(t) \rightarrow+\infty \quad \text { y } \psi^{\prime}(t)=\frac{1}{b\left(\frac{1}{t^{p^{\prime}}}\right)} \rightarrow+\infty \quad \text { cuando } t \rightarrow+\infty
$$

Definamos

$$
\phi(t):=\psi^{-1}(t), \forall t \geq 1
$$

Entonces $\phi$ es una función $C^{2}$ cóncava y estrictamente creciente, satisfaciendo

$$
\phi(t) \rightarrow+\infty \quad \text { y } \quad \phi^{\prime}(\psi(t))=\frac{1}{\psi^{\prime}(t)} \rightarrow 0 \text { cuando } t \rightarrow+\infty
$$

Así $\phi$ tiene todas las propiedades que usamos para conseguir (4.7) y (5.16). Usando $\rho(t)=b^{-1}(\phi(t))$ 
y haciendo un cambio de variables $t=\psi(\tau)$, usando (5.19) y haciendo un otro cambio de variable $\tau=\phi(t)$, y usando $b^{-1}\left(\frac{1}{\psi^{\prime}(\tau)}\right)=b^{-1}\left(b\left(\frac{1}{\tau^{p^{\prime}}}\right)=b^{-1} \circ b\left(\frac{1}{\tau^{p^{\prime}}}\right)=\tau^{-p^{\prime}}\right.$ obtenemos

$$
\begin{aligned}
\int_{1}^{+\infty} \rho(t)^{\frac{1}{p}} \phi^{\prime}(t) d t & \left.=\int_{1}^{+\infty}\left[b^{-1}\left(\phi^{\prime}(t)\right)\right]^{\frac{1}{p}} \phi^{\prime}\right) d t \\
& =\int_{1}^{+\infty}\left[b^{-1}\left(\phi^{\prime}(\psi(\tau))\right)\right]^{\frac{1}{p}} d \tau \\
& =\int_{1}^{+\infty}\left[b^{-1}\left(\frac{1}{\psi^{\prime}(\tau)}\right)\right]^{\frac{1}{p}} d \tau \\
& =\int_{1}^{+\infty} \frac{1}{\tau^{\frac{p^{\prime}}{p}}} d \tau \\
& =\lim _{M \rightarrow+\infty} \frac{1}{\frac{p^{\prime}}{p}+1}\left\{M^{-\frac{p^{\prime}}{p}+1}-1\right\} \text { y desde que }-\frac{p^{\prime}}{p}+1<0 \\
& =\frac{1}{\frac{p^{\prime}}{p}-1}>0 .
\end{aligned}
$$

\section{Estimativa dependiendo de $\phi$}

Desde que $\psi \circ \phi=I$ entonces $\psi^{\prime}(\phi(t)) \phi^{\prime}(t)=1$, y luego $\phi^{\prime}(t)=\frac{1}{\psi^{\prime}(\phi(t))}=b\left(\frac{1}{\phi(t)^{p^{\prime}}}\right)$, de donde deducimos

$$
\rho(t)=b^{-1}\left(\phi^{\prime}(t)\right)=b^{-1} \circ b\left(\frac{1}{\phi(t)^{p^{\prime}}}\right)=\frac{1}{\phi(t)^{p^{\prime}}} .
$$

Por otro lado, usando (5.20), obtenemos

$$
\begin{aligned}
\int_{S}^{T} \rho(t)^{\frac{1}{p}} \phi^{\prime}(t) d t & =\int_{S}^{T} \frac{1}{\phi(t)^{\frac{p^{\prime}}{p}}} d t \\
& =\frac{1}{1-\frac{p^{\prime}}{p}}\left[\phi(t)^{1-\frac{p^{\prime}}{p}}\right]_{S}^{T} \\
& =\frac{1}{1-\frac{p^{\prime}}{p}}\left\{\phi(t)^{1-\frac{p^{\prime}}{p}}-\phi(S)^{1-\frac{p^{\prime}}{p}}\right\} \\
& =\frac{1}{\frac{p^{\prime}}{p}-1}\left\{\phi(S)^{1-\frac{p^{\prime}}{p}}-\phi(T)^{1-\frac{p^{\prime}}{p}}\right\} \\
& \leq \frac{1}{\frac{p^{\prime}}{p}-1}\left\{\phi(S)^{1-\frac{p^{\prime}}{p}}\right\} \text { puesto que } \phi=\psi^{-1}>0 .
\end{aligned}
$$


Usando (5.20) y (5.21) en (5.16) tenemos

$$
\begin{aligned}
\int_{S}^{T} E(t)^{\sigma+1} \phi^{\prime}(t) d t & \leq C E(S)^{1+\sigma}+E(S) \frac{C}{\phi(S)^{\frac{p^{\prime}}{p}}}+E(S) \frac{C}{\phi(S)^{\frac{p^{\prime}}{p}-1}} \\
& \leq C E(S)^{1+\sigma}+E(S) \frac{C}{\phi(S)^{\frac{p^{\prime}}{p}-1}} .
\end{aligned}
$$

Entonces aplicando el Lema 2.1 desde que (2.7) sucede con $\sigma^{\prime}=\frac{p^{\prime}}{p}-1>0$, y deducimos que existe una constante $C$ dependiendo continuamente de $E(1)$, tal que

$$
E(t) \leq \frac{C}{\phi(t)^{\frac{p^{\prime}}{p^{\sigma}}}}=\frac{C}{\phi(t)^{\frac{p^{\prime}}{\theta}}} \forall t \geq 1
$$

\section{Crecimiento de $\phi$}

Estimar el crecimiento de $\phi$ es equivalente a acotar la función $\phi^{-1}=\psi$.

Sea $T_{0}$ tal que $b\left(\frac{1}{\tau^{p^{\prime}}}\right) \leq 1, \quad \forall \tau \geq T_{0}$.

Si $s<\tau$ desde que $b$ es creciente tenemos $b\left(\frac{1}{\tau^{p^{\prime}}}\right) \leq b\left(\frac{1}{s^{p^{\prime}}}\right)$, i.e. $\frac{1}{b\left(\frac{1}{s^{p^{\prime}}}\right)} \leq \frac{1}{b\left(\frac{1}{\tau^{p^{\prime}}}\right)}$.

Por otro lado, tenemos que: si $p^{\prime} \geq 1$ se verifica $1+(\tau-1) z \leq \tau^{p^{\prime}} z$ para $z \geq 1$ y $\tau \geq 1$. En efecto, basta probar que $1 \leq\left(1-\tau+\tau^{p^{\prime}}\right) z$. Si $\tau \geq 1$ entonces $\tau^{p^{\prime}}-\tau \geq 0$ y así $1+\tau^{p^{\prime}}-\tau \geq 1$; luego multiplicando por $z \geq 1$ tenemos $\left(1-\tau+\tau^{p^{\prime}}\right) z \geq 1$.

Usando estas observaciones obtenemos

$$
\begin{aligned}
\psi(\tau) & =1+\int_{1}^{\tau} \frac{1}{b\left(\frac{1}{s^{p^{\prime}}}\right)} d s \leq 1+\frac{1}{b\left(\frac{1}{\tau^{p^{\prime}}}\right)} \int_{1}^{\tau} d s \leq 1+(\tau-1) \frac{1}{b\left(\frac{1}{\tau^{p^{\prime}}}\right)} \\
& \leq \tau^{p^{\prime}} \cdot \frac{1}{b\left(\frac{1}{\tau^{p^{\prime}}}\right)}=\frac{1}{B\left(\frac{1}{\tau^{p^{p^{\prime}}}}\right)}
\end{aligned}
$$

Luego, haciendo $t=\frac{1}{B\left(\frac{1}{\tau^{p^{p^{\prime}}}}\right)}$ (esto es $\frac{1}{\tau^{p^{\prime}}}=B^{-1}\left(\frac{1}{t}\right)$ ) y usando (5.24) tenemos $\psi(\tau) \leq t$, de donde tenemos $\tau \leq \psi^{-1}(1)=\phi(t)$, esto es

$$
\frac{1}{\phi(t)} \leq \frac{1}{\tau}
$$


Así, usando (5.25) en (5.23) obtenemos

$$
E(t) \leq \frac{C}{\phi(t)^{\frac{p^{\prime}}{p}}} \leq \frac{1}{\tau^{\frac{p^{\prime}}{\theta}}}=\left(B^{-1}\left(\frac{1}{t}\right)\right)^{\frac{1}{\theta}},
$$

donde $\theta=\frac{N}{2 m}$.

\section{REFERENCIAS BIBLIOGRÁFICAS}

[1] Conrad F. and Rao B. Decay of solutions of wave equations in a star-shaped domain with nonlinear boundary feedback. Asympt. Anal. 7, 159 - 177. (1993).

[2] Cortés L. A note on resonant frequencies for a system of elastic wave equations. Int. J. Math. Sci. 64, 3485 - 3498. (2004).

[3] Ikawa M. Mixed problems for hyperbolics equations of second order. J. Math. Soc. Japan 20, 580 - 608. (1968).

[4] Kesavan S. Topics in Functional Analysis and applications. John Wiley \& Sons, (1989).

[5] Pazy A. Semigroups of Linear Operator and Applications to Partial Differential Equations, Springer, New York, (1983).

[6] Komornik V. Exact controllabity and stabilization. John Wiley \& Sons. (1994).

[7] Martinez P. Decay of solutions of the wave equation with a local highly degenerate dissipation. Asymptotic Analysis 19, 1 - 17. (1999).

[8] Nakao M. Decay of solutions of the wave equation with a local degenerate dissipation. Israel J. Of Maths 95, P, 25-42. (1996).

[9] Santiago Y. A. Una aplicación del Lema de Nakao. Revista de los Departamentos de la Facultad de Ciencias Matemáticas, UNMSM, Nro. 2 (2006).

[10] Santiago Y. A. Decaimiento exponencial de la solución débil de una ecuación de onda no lineal. PESQUIMAT Revista de la Fac. CC. MM. de la UNMSM. Vol VIII Nro. 2, Diciembre, pág 29 - 43. (2005).

[11] Santiago Y. A. and Rivera J. Global existence and exponential decay to the wave equation with localized frictional damping. PESQUIMAT Revista de la Fac. CC. MM. de la UNMSM. Vol. V. Nro. 2, Diciembre, pág. 1 - 19. (2002).

[12] Santiago Y. A. Algunas desigualdades integrales y aplicaciones. (2006).

[13] Zuazua E. Exponential decay for the semi-linear equation with locally distributed damping. Comm. Partial Differential Equations 15, 205-235. (1990). 